\title{
Entwicklung und Bedeutung einer transkulturellen Grundhaltung als (Körper-)Psychotherapeut am Beispiel China
}

\section{Ein ethnoanalytischer Essay}

\author{
Ulrich Sollmann \\ Psychotherapie-Wissenschaft 8 (2) 21-28 2018 \\ www.psychotherapie-wissenschaft.info \\ CC BY-NC-ND \\ https://doi.org/10.30820/8243.03
}

\begin{abstract}
Zusammenfassung: Kollegen in China körperpsychotherapeutische Konzepte vorzustellen, stellte sich für den Autor als ein ethnologischer Erfahrungsprozess dar. Die Teilhabe am Geschehen im jeweiligen (persönlich, beruflich, privat) Lebensfeld gestaltete sich als Körper-zu-Körper-Kommunikation. Hierdurch impliziert nutzte der Autor einen heuristischen Zugang insoweit, als er entsprechend einer professionellen Naivität zunächst über den Prozess des EntLernens (eigener kultureller Vorverständnisse) ein auf Sinneserfahrung basierendes «Wissen» zu generieren bemüht war, das vor allem auch auf der sensorischen, emotionalen sowie transkulturellen Kommunikation basierte. Insoweit war der Prozess prinzipiell offen. Um einen solchen Prozess des «sensed knowing» wirkungsvoll, plastisch, aber auch professionell wiederzugeben, scheint dem Autor zu diesem Zeitpunkt das Mittel der Narration geeignet zu sein, sprich die Form eines professionellen Essays. In dem Beitrag erfährt man einerseits etwas über das Zusammenspiel in China von Struktur, Lebensfeldern, sowie der bunten, vielschichtigen, dynamischen, eigenwilligen Selbstregulation der Menschen vor Ort. Andererseits bietet der Artikel Einblicke in den ethnologischen «Instrumentenkoffer», der bei der transkulturellen Kommunikation hilfreich, sinnvoll und praktisch nutzbar sein kann.
\end{abstract}

Schlüsselwörter: Körperpsychotherapie, China, Ethnologie, Narration, Verhaltensmuster, transkulturelle Kommunikation

\section{Prolog}

Meine Erfahrungen als Körperpsychotherapeut in China könnten in einem Fachartikel zusammengefasst werden. In einem solchen könnte ich wesentliche Aspekte transkultureller Erfahrung und Kommunikation, so wie sie mir in den letzten Jahren begegnet sind, darstellen und fokussiert diskutieren. Je mehr ich drüber nachdenke, desto schwieriger stelle ich mir ein solches Unterfangen vor. Ich kann mich nämlich noch gut an die recht chaotischen Erlebnisse meiner ersten Aufenthalte in China erinnern. Völlig unerwartet musste ich mir das Versagen meiner sonst so erprobten kulturellen Orientierung eingestehen. Ent-Lernen (Simon, 2010 [1997]) war angesagt. Es bedarf zunächst des Ent-lernens, das heißt der Blockierung von irgendwie erfolgreichen und das Überleben bisher sichernden Strategien, um neue Muster lernen zu können. Desorientierung begleitete mich daher anfangs Tag für Tag. Kommunikation war einfach nicht mehr das, was und vor allem wie ich es gewohnt war. Und dabei wollte ich doch nur, wohl voller Begeisterung für das erhoffte Neue, meinen Job verantwortlich machen.

Ich erlebte mich radikal und gnadenlos auf mich selbst zurückgeworfen, ohne sinnvolles Erfahrungs-Know-How, ohne tragfähiges professionelles Wissen um die chinesische Kultur. Zurückgeworfen auf das in mir ständig belebte, unbekannte, emotionale Echo, einer Resonanz gleich, das mich und alle meine Sinne erschütterte. Dies ist vergleichbar mit der von Lorenzer (1972) beschriebenen Ambiguitätstoleranz. Es blieb mir also nichts anderes übrig, als mich auf mein körperinneres Sensorium, die Körper-zu-Körper-Kommunikation jeweils vor Ort und einen, man könnte sagen, ethnologischen «Welpenschutz» verlassen. Hierunter verstehe ich: sich spielerisch, relativ absichtslos zu verhalten, indem man sich auf die Suche nach erlebbarem, erlebtem Verstehen macht. Dies ist insoweit absichtslos, als man sich (noch) nicht unter einer spezifischen (akademischen, wissenschaftlichen) Fragestellung auf den Weg der teilnehmenden Beobachtung begibt. Eine so verstandene Feldforschung ist gerade zu Anfang heuristisch. Ein phänomenologisches Vorgehen scheint angezeigt zu sein. Die Kommunikation eines solchen ethnologischen Projekts gelingt, zumindest zu Beginn, am ehesten in narrativer Form. Bietet doch ein solches Vorgehen eine sinnvolle, konkrete und relevante Möglichkeit, das transkulturelle Zusammenspiel von beobachtetem Phänomen, eigener Beteiligung und beginnender Entwicklung von professionellen Kriterien zu würdigen.

Erkenntnisleitendes Interesse ist die Überzeugung, dass nicht nur der Fremde fremd ist, sondern in jedem Kulturkreis ich auch fremd bin. Die Relevanz meines Beitrags für die Arbeit als Psychotherapeut im transkulturellen Bereich besteht somit in dem Erwerb einer Grundhaltung, die auf 
der spezifischen Erfahrung des «sensed knowing» basiert. Diese zu beschreiben, mich im Prozess dieses Erwerbs, mich als Prozess zu beschreiben, ist eine wesentliche Aufgabe meines Beitrags. Vergleichen kann man dies mit dem Soundcheck vor einem grossen Konzert, durch den das Zusammenspiel zwischen Musikern, Instrumenten und Publikum in einer bestimmten Örtlichkeit erst zu einem Hör- und Erlebensereignis wird.

\section{Körper-zu-Körper-Kommunikation ist (auch) Ent-Lernen}

Der Verlust des Vertrauten warf mich gerade zu Anfang regelmässig in einen Strudel des (Ent-)Lernens. Ich spürte hautnah das, was nicht mehr funktionierte, was für mich keine sensorisch-emotionale Gültigkeit mehr hatte. Dieser Strudel liess mir keine Chance zum Durchatmen, noch hätte ich emotionale Rettung in der Anleihe an von China-Experten vielfach gepriesenen Tipps und Tricks gefunden, wie man sich in China sicher bewegen könne. Der ungewollte, spontane Sprung ins Ungewisse liess mir keine Zeit der Reflektion, der hinreichenden Selbstkontrolle oder eines emotionalen Abgleichs mit ähnlichen Situationen.

Direktes Handeln und unmittelbares Reagieren waren angesagt. Handeln war in diesem Falle schlichtweg Körperhandeln, Agieren oder Reagieren. Das, was ich bisher in meinem Leben gelernt hatte, bot mir in China nur rudimentär Verhaltenssicherheit. Befreit von zu viel kognitiver (Selbst-)Kontrolle begann ich auch die Freiheit und Lust zu geniessen, einfach zu machen, was mir spontan in den Sinn kam oder wohin der Fluss des Alltags mich hintrieb, und zu verfolgen, wie ich auf Gegensätze reagierte. Gerade letzteres stellte sich als kognitive Herausforderung dar. Bin ich doch eher als Kind einer die kognitive (Selbst-) Kontrolle favorisierenden Kultur geboren geworden.

Meine Erfahrung in China forderte stets meine kognitive Selbstkontrolle heraus: einerseits die Kontrolle in mir und meinem eigenen Wahrnehmungs- und Erlebensapparat gegenüber, andererseits die Kontrolle in der jeweiligen Situation, wenn nämlich ganz überraschend etwas auftaucht, was meinem bis dahin existierenden Vorverständnis von China nicht entspricht. Northoff (2015) hat sehr plastisch beschrieben, wie Kultur Wahrnehmung prägt und auch umgekehrt Wahrnehmung Kultur schafft.

Ich möchte hier auf die Juxtaposition, einen interessanten Fachbegriff aus der Sprachwissenschaft verweisen. Gemeint ist damit die Zusammenfügung zweier Wörter, die in dieser spezifischen Koexistenz etwas Drittes meinen. So setzt sich zum Beispiel «Fussballspiel» aus den beiden Wörtern «Fussball» und «Spiel» zusammen, die für sich genommen zwei unterschiedliche Dinge bezeichnen, zusammengefügt als «Fussballspiel» aber eine neue Bedeutung, etwas Drittes ergeben. Bezogen auf das Erleben einer Szene im jeweiligen Lebensfeld geht es dann darum, erlebbare Dinge miteinander zu vereinen, auch wenn sie aufgrund des Gegensatzes nicht vereinbar sind. «Für Ricoeur ist es gerade die Spannung des Gegensatzes, aus der man heraus eine besonders kraftvolle Position herstellen kann, im Gegensatz zu Hegels Dialektik, die durch Synthese der gegensätzlichen Pole die Aufhebung letzterer bewirkt» (Leonard, 2018).

Hierzu folgt ein Beispiel in Bezug auf China: China ist dafür bekannt, das Internet zu kontrollieren, bestimmte Seiten und Inhalte zu blockieren. Dies ist ein Verhaltensmuster - nämlich offizielle Politik - der Zentralregierung in Peking. Demgegenüber gibt es zeitgleich eine offizielle Order aus Peking an die Regionalregierungen, die User im Netz als Kunden zu verstehen und zu bedienen. Man solle doch sehr sorgsam mit den Selbstäusserungen, Interessen und Wünschen besagter Kunden umgehen und diesen entgegenkommen. So gesehen gibt es Internetkontrolle und Nichtkontrolle zugleich. Es geht mir nicht um die Klärung der Frage, ob es Kontrolle gibt oder nicht, sondern es geht mir darum, was aus dem Vorhandensein von Kontrolle und Nichtkontrolle zugleich «geboren» wird. Die Geburt des «Dritten» im Sinne von psychosozialer Induktion (Klüwer, 1974) hängt massgeblich auch von mir als Beobachter ab, der das Zusammenspiel von Kontrolle und Nichtkontrolle auf eine jeweils sehr spezifische, persönliche szenische Art und Weise erlebt und deutet, um sich der Deutung entsprechend zu verhalten. Das Überraschungsmoment ist also ein wesentlicher Bestandteil der Juxtaposition auf der Erlebensebene. (Ent-)Lernen meint daher stets auch, offen zu sein für eine derartige Erfahrung.

Die Spontaneität und Intensität des Erlebens liessen mich oftmals die gewaltige Kraft spüren, die von der Erfahrung der «Vereinbarung des Unvereinbaren» ausgeht (vgl. Ricoeur 1973). Eine machtvolle Kraft, dass es so und nicht anders ist. Ich reagierte auf sie, ohne zu warten, ohne reflektieren oder mich erst einmal mit dem Gegenüber austauschen zu können. Ich habe dies im Grossen und im Kleinen erlebt.

\section{Vom Überleben im Gewusel}

Die Überraschungen in solchen Erfahrungsszenen weckten natürlich auch eine Neugier darauf, was denn nun alles (auch in mir) passieren, wie es mir in Zukunft ergehen und wie ich mit einem solchen Geschehen umgehen würde. Wie gesagt, ich wollte mich durch solche Herausforderungen nicht unterkriegen lassen und spürte somit auch eine Lust, mich selbst zu behaupten, mich selbst zu fordern, eben nicht vor überraschenden Herausforderungen zu kneifen. Chinesen besitzen, so scheint mir, einen solchen Überlebenswillen und eine entsprechende Findigkeit, diesen nicht zu verlieren. Einerseits lassen sie sich dabei nicht in die eigenen Karten schauen: Man bleibt im Strom oder dem Gewusel der Menschenmassen recht angepasst und unauffällig. Andererseits, und das könnte wie ein Gegensatz scheinen, macht man das, was man gerade machen will: so zum Beispiel die Frauen mittleren Alters, die, trotz heftiger Proteste in den Medien, tagsüber irgendwo ihre Musikbox auspacken, um selbstbewusst, für jedermann sichtbar, die Praxis ihrer Gymnastikübungen zu pflegen. 


\section{Professionelle Naivität der Erfahrung}

Da ich primär nicht als Tourist in China war, lag es nahe, stets meine «Forscherbrille» aufzusetzen. Gepaart mit der nötigen Entdeckerfreude begann ich also, Begegnungsszenen und Kontaktversuche zwischen Chinesen und mir wie ein Kind mit offenen Sinnen zu erleben und zu betrachten. Was passierte da? Wie begegnete mir das, was geschah? Und wie entwickelte es sich? Was geschah, wenn sich die Blicke zwischen einem Chinesen und mir trafen? Wortlos diesen überraschenden Augenblick erfassend? Ich erinnere mich an einen meiner Lehrer, der uns in Zeiten der Wissensvermittlung an der Universität eine zusätzliche Tür des heuristischen Erkennens aufzeigte. Ihm sei es manchmal wichtiger, eine neue Frage zu finden als sich einer neuen Erkenntnis hinzugeben, einer Antwort, die, wie mir heute scheint, eher wie ein flüchtiger Windhauch zur wissenschaftlichen Bequemlichkeit führen kann, zu einer wiederkehrenden (Selbst-)Bestätigung von etwas, was man vielleicht ohnehin schon wusste.

Fragen wirken wie das Gewürz beim Kochen, durch das man den Eigengeschmack der Zutaten hervorherben, fördern und überhaupt erst in seiner Gänze entdecken kann, die aber auch bei leichter Überdosierung das ganze Essen verderben können. Die ersten Schritte in meinem privaten Forschungsprojekt waren gekennzeichnet durch learning by doing, learning by walking, learning by looking, listening, tasting. Ich hatte inzwischen den Zustand des unvorbelasteten, spontanen Erlebens auch als Chance geniessen gelernt, einfach einmal nichts zu tun und die Dinge absichtslos zu betrachten. Völlig erleichtert erlebte ich mich wie in einem Zwischenraum zwischen deutscher und chinesischer Kultur, zwischen berührbarem, aber noch fremdem China sowie dem nicht mehr sicheren, aber auch überflüssigen Deutschen in mir. Ich sah mich nicht mehr gedrängt, als Deutscher vornehmlich gezielt wahrzunehmen oder kulturelle Unterschiede in ihrer Bedeutung absichtsvoll zu registrieren, noch sah ich mich genötigt, "China» und "die Chinesen» verstehen zu müssen, um ihnen «kulturell» entsprechend» zu begegnen. Dieser Zwischenraum lud mich zu einer unvorbelasteten Naivität der Erfahrung ein, einem Kind gleich, das sich in einer neuen (Spiel-)Umgebung befindet, die es jetzt - für Aussenstehende chaotisch anmutend - «bespielt»:

Mal greift es nach diesem Gegenstand, mal spielt es mit jenem, mal geht es zurück zu einem Gegenstand, mit dem es schon gespielt hat, ohne aber zielgerichtet, ohne zu etwas Bestimmten getrieben, sondern in professioneller Naivität der Erfahrung intentional Dinge anzufassen und dort zu verweilen.

\section{«Ethnologischer Welpenschutz»}

Ich genoss einen «ethnologischen Welpenschutz», erlaubte mir eine professionelle "Narrenfreiheit», indem ich mich in unbewusster Vorahnung der Erkenntnis, dass der Weg das Ziel sei, wie auf einer Eisscholle auf dem Meer treiben liess - mal angetrieben durch den Wind, mal gezogen durch eine Strömung, mal abgestossen durch die Berührung mit einer anderen Eisscholle.

Indem ich mich für die alltagsweltlichen Ausdrucksformen, so wie sie mir jeweils situativ, szenisch begegneten, interessierte, wurde mir klar, dass ich mich mit dem in den Sozialwissenschaften beschriebenen Raum, Lebenswelt genannt, bewegte. Indem ich so, wie ich es tat, bemüht war, an dem mir jeweils konkret beobachtbaren und erfahrbaren Leben (Lebensszene) teilzunehmen, spielte ich mit einer mir beruflich und persönlich wichtigen Umgangsweise, nämlich der Annäherung - um eben teilzunehmen, gleichzeitig zu beobachten und mir Notizen machen zu können (Plessner, zit.n. Honer, 2008, S. 198). Es war mir wichtig nicht (nur) klassisch wissenschaftlich zu beobachten und (quantitativ) Fakten zu erfassen, sondern die Welt meines Gegenübers mitzuerleben, mitzuerfahren und in einer dichten Beschreibung (Geertz, 2003) zu erfassen. Mir ging es nicht um wissenschaftliche Faktenerhebung, sondern um den Beginn von Erspüren, von Erfahren, von Begehen, von Teilhaben und von beginnender Kundigkeit. Gerade diese Art des Vorgehens ermöglichte es mir, emotional, sensorisch und mental offen zu sein, offen zu bleiben, zunehmend erfahrener zu erleben und mich durch Abenteuer und "Experimente», so wie sie mir an der nächsten Ecke begegnen konnten, überraschen zu lassen. Manchmal ertappte ich mich bei der Vorstellung, meine Erfahrungen und Eindrücke reportageartig niederzuschreiben. Anfangs erlebte ich mich noch in dieser Rolle, um mich bald eher im Sinne eines «nosing-around» (vgl. Lindner, 2004) in der fremden Kultur zu bewegen. Man kann sich dies wie einen Wabrnehmungsspaziergang vorstellen, bei dem ich mal hier, mal dort im wahrsten Worte herumschnüffelte. Angetrieben wurde ich dabei von der kindlich wirkenden Naivität in Bezug auf das Neue, aber auch der wiederkehrenden Zurücknahme durch eine grundsätzliche Skepsis, wenn ich mir nämlich Notizen machte und mich bewusster in diesem Übergangsraum zwischen meiner eigenen und der chinesischen Kultur erlebte.

\section{Mitten im Bedeutungsgewebe}

Das sich mir auftuende Bedeutungsgewebe gestaltete sich anfangs noch sehr geheimnisvoll, als eine Bühne, auf der wir, mein chinesisches Gegenüber und ich, gemeinsam den Akt in einem schier endlosen Theaterstück spielten. Ich berühre mein Gegenüber dabei in einem haptisch erfahrbaren Raum. Darunter verstehe ich erweitert das, was man berühren, schmecken, riechen, hören kann. So wie ein Vogel an einem vorbeifliegen mag, begann ich Relevanzen zu vermuten, die sich jeweils für einen winzigen Moment vor meinem inneren Auge auftaten, die mögliche Relevanz meines Gegenübers von dem, was er tat, wie er mich anschaute oder mich ignorierte. Ich stolperte immer wieder in die nächste Verunsicherung. Glaubte ich dort, wo ich gerade war, mich für einen Moment ausruhen und durchatmen zu können, brach schon wieder etwas Neues auf, eine neue Erfahrung, eine Begegnung oder aber auch 
der Abbruch eines Kontakts, der mich für einen Moment Sicherheit hatte spüren lassen. Wie im Flug reihten sich solche völlig unzusammenhängenden Erfahrungsszenen aneinander, wie in einem Film oder Theaterstück, das auf einer Szenenfolge aufbaut, deren Sinn, Zusammenhang und Botschaft sich gerade anfangs überhaupt noch nicht erschliessen lassen.

Die Reziprozität der Perspektiven bezog sich aber auch auf den Innenraum meines Erlebens, meines Denkens und Reflektierens. Mich einzulassen hiess nämlich immer, auch mich selbst zu reflektieren, mir selbst gegenüber immer wieder Rechenschaft abzulegen, welchen Part ich denn in dem Moment spielen würde, wie und warum ich jetzt die Dinge so erleben und sehen würde und nicht anders. Ungefragt Teil dieser fliessenden gemeinsamen Bewegung zu sein, half mir, mich zunehmend, wenn auch oftmals stolpernd und unbeholfen, anfangs nur rudimentär orientieren zu können. Dies war natürlich keine Orientierung im Sinne von «Ich weiss jetzt Bescheid. Ich weiss, warum mein Gegenüber das so und nicht anders macht», sondern es war eine sensorische, emotionale, mentale Orientierung mich überhaupt in diese Art der Erkundung, die Feinheit des Erspürens einer derart neuen Lebenswelt fallen lassen zu können - unbedarft, naiv, begierig, mutig, manchmal zum Glück wagemutig.

\section{Die Bedeutung des körperinneren Sensoriums}

Eine meiner ersten und wichtigsten Erfahrungen und neuen Erkenntnisse betrifft das Vertrauen in das eigene körperinnere Sensorium. Hierunter verstehe ich das Ensemble aller Sinneseindrücke, Insbesondere auch das Körpererleben als einer eigenständigen Perspektive von Wahrnehmung. Mit Geuter (2015, S. 24) betrachte ich «den Körper als den personal erlebten Körper eines lebendigen Subjekts». Schatz (2002, insb. S. 77f.) nennt sieben verschiedene Ebenen oder Perspektiven von Körpererleben. Sie im Einzelnen auszuführen, würde den Rahmen dieser Arbeit sprengen, sie sollen an dieser Stelle aber kurz zusammengefasst werden:

$>$ Der objektive Körper umfasst den medizinisch-biologischen Körper.

$>$ Der subjektiv erlebte oder ausgedrückte Körper meint den Körper aus individueller Perspektive.

> Der im Raum bewegte Körper meint den Körper, der sich bewegt und/oder im Raum geht, springt, läuft usw.

$>$ Der Körper trägt natürlich immer auch Vergangenheit, Gegenwart und Zukunft in sich. Die hiermit verbundenen Erfahrungen machen den chronologischen Körper aus.

$>$ In Beziehung zu einem anderen Menschen verwirklicht sich der relationale Körper.

> «Überlässt» man sich dem Körper, spricht man vom autonomen Körper. Das Körpererleben ist dann eher geprägt durch autonome, nicht kontrollierte Ausdrucksbewegungen, spontanes Gefühl sowie die Reizung des Körperinneren als einem eigenständigen Sinnesorgan.
$>$ Schliesslich gibt es die schöpferische, kreative Perspektive des Körpers, wenn er sich schöpferisch, künstlerisch, zum Beispiel über Tanz, Pantomime, Musik usw. ausdrückt.

Immer fungiert der Körper dann als ein eigenständiges Sinnesorgan. Die hierdurch gespürten oder erlebten Eindrücke dringen nur langsam in mein Bewusstsein. Sie begleiten mich jedoch unbewusst bei jedem Schritt. Beim Betreten einer fremden Kultur und der spontanen und überraschenden Begegnung mit den Menschen wähnte ich mich als körperpsychotherapeutischer Ethnologe mit einem differenzierten, funktionierenden und kritischen Wahrnehmungsapparat sowie der entsprechenden sensorischen Offenheit für das Neue, das Fremde gut ausgestattet. So hoffte ich, offen, frei und sensibel genug für neue Eindrücke zu sein. Ich erlebte unmissverständlich und stets aufs Neue, dass das so, wie ich es bisher gewohnt war, aber nicht funktionierte.

Mir wurde klar, wie essenziell wichtig es ist, sich zunächst mit der Art der Wahrnehmung, dem Wie der neuen Wahrnehmung auseinanderzusetzen, um hierdurch die Grundausrüstung und einen tragfähigen Erfahrungsboden zu entwickeln, der gewissermassen das Handwerkszeug bietet, sehen, hören, riechen, berühren zu können und um Menschen zu begegnen und später - das war ja mein eigentliches Anliegen als Körperpsychotherapeut - entsprechend mit ihnen arbeiten zu können.

Die Erforschung des eigenen körperinneren Sensoriums und die Regulierung der sensorischen Wahrnehmung überhaupt gelingt, so meine feste Überzeugung, schliesslich nur im Austausch mit jemandem aus der fremden Kultur. Nazarkiewicz und Krämer (2012) nennen dies transkulturelle Kommunikation.

\section{Körper und Schamerleben}

Eine erschütternde diesbezügliche Erfahrung machte ich bei einer Präsentation mit Kollegen auf einem Kongress in Shanghai. Ich wurde gebeten, etwas über die Bedeutung von Körpersprache und nonverbaler Kommunikation zu erzählen. Zur Illustration bat ich eine sich freiwillig meldende Teilnehmerin nach vorne, um ihr eine kleine Übung zu erläutern.

Sie sollte die Augen schliessen. Der Abstand zwischen ihr und mir betrug etwa zwei Meter und ich versicherte ihr, dass ich sie nicht berühren würde. Ich wollte durch die Übung zeigen, dass der Körper immer reagiert, sobald ein Impuls von aussen kommt (in diesem Fall sollte es sich um einen auditiven Impuls handeln), auch wenn man sich selbst kontrollieren möchte. Die Teilnehmerin hielt also die Augen geschlossen. Ich klatschte nach einer Weile einmal in die Hände, sodass sie ein wenig erschrak. Die Schreckreaktion war natürlich körperlich sichtbar. Nachdem sie die Augen wieder geöffnet hatte, bat ich sie zunächst nur, die spezifische Reaktion in ihrem Körper zu beschreiben. Sie erzählte direkt, klar und ausführlich sowie persönlich genau, was sie in ihrem Körper 
wahrgenommen hatte: Sie berichtete von Körpersensationen in den Beinen, im Nacken, in den Augen und im Bauchbereich. Dabei unterstrich sie jeweils gestisch das, was sie sagte. Natürlich zeigte sie auch auf ihren BauchBeckenbereich, berührte diesen Bereich, hörte dabei aber spontan und überraschend auf zu reden. Ich registrierte die leichte Verzögerung und Irritation, wandte mich daher an die Übersetzerin, die genauer zu verstehen bemüht war, was die Chinesin ausdrücken wollte. Aber auch die Übersetzerin verlor ihre Worte.

Es war ziemlich klar, wo im Körper die Teilnehmerin etwas gespürt hatte. Sie schien ihren eigenen BauchBeckenbereich auch (un-)bewusst zu fühlen. Ihre Hände berührten ihren Bauch-Beckenbereich und, so vermutete ich, sie fühlte auch die mit diesem Bereich verbundenen Emotionen - wenn auch vermutlich kulturell bedingt unerlaubt -, ohne sie mit Worten ausdrücken zu können. Ich vermute, dass sie auf nonverbaler Ebene durch ihre Geste nicht nur den Körper als solchen berührte, sondern auch als einen körperlich-symbolischen, kulturell-emotionalen tabuisierten Raum. Ist er doch auch unbewusst Ort von gefühlter oder fantasierter Intimität sowie Sexualität. Unbewusst emotional aus dem Lot gebracht, verlor sie regelrecht ihre Sprache für etwas, was körperlich präsent war und was sie mit ihren eigenen Händen intentional berührt hatte, wollte sie uns doch durch Worte und Gesten von dem berichten, was sie im Körper empfunden hatte.

Dieses Geschehen war insoweit kommunikativ und kulturell «ansteckend», als die Übersetzerin just in dem gleichen Moment ihre Sprache verlor - eine Frau, die mit der Übersetzung von körperbezogenen Themen vertraut ist. Natürlich war ich bemüht, dem wortlosen Geschehen respektvoll Raum zu lassen, ohne dass sich eine der beiden Chinesinnen verbal «entblössen» musste. Daher beruhigte ich die beiden Frauen mit dem Hinweis, sie bräuchten jetzt nicht weiter zu sprechen, wenn sie es so wollten. Auch erzählte ich von meinem Eindruck, dass durch diese kleine Demonstrationsübung bereits ein Prozess des sehr persönlichen Erlebens in Gang gesetzt worden war, der einen respektvollen Umgang erforderte. Ebenso beleuchtete ich kurz die vierfache Wirkung auf die Kollegin: Erstens konnte ihre Selbstkontrolle die Wirkung der Geräusche auf ihren Organismus nicht verhindern; zweitens blieb ein Teil der Körperreaktion, des Körperempfindens emotional unbewusst; drittens berührt immer auch das Körpererleben die mit diesem Körperbereich verbundene emotionale Welt; viertens hat dies immer eine Auswirkung auf das zwischenmenschliche Geschehen.

Gleichzeitig wandte ich mich an die anderen Workshop-Teilnehmer, um sie durch meine Ausführungen einzubeziehen. Ich ahnte, dass die anderen Gruppenteilnehmer eine entsprechende kulturell-emotionale Resonanz spürten, hatte ich doch während des Geschehens ihr zustimmendes Kopfnicken gesehen.

Mir schien, dass die Chinesin, die die Übung durchführte, meine Übersetzerin und ich trotz der Wortlosigkeit sowie der impliziten emotionalen Berührung im Erleben von Scham und Intimität in gutem, res- pektvollem Kontakt miteinander waren. Daher lag mir daran, diese Beziehungsszene durch die vorschnell von mir befürchteten, als Abwehr verstandenen Fragen der anderen zu bremsen. Es war wichtig - und das betonte ich, indem ich mich an alle richtete -, sich auch gerade für die Wortlosigkeit im gemeinsamen Kontakt, in der «guten Beziehungsszene», Zeit zu nehmen, ohne sich kompensatorisch oder gar abwehrend «verbal zu eilen». Zur Bewusstmachung und emotionalen Sicherung dieser Erfahrung gehört auch die Verbalisierung von Wortlosigkeit und die der Bedeutung von Wortlosigkeit als einem Erleben von Kontakt, Intimität und Scham. Die Chinesin fühlte sich inzwischen sichtlich besser, fand wieder zurück zu ihrer Sprache, sodass wir die Demonstrationsübung gemeinsam beenden konnten. Um das Geschehen, den Situationsraum didaktisch zu klammern, wandte ich mich daher nun an die restlichen Teilnehmer des Workshops. Erleichtert reagierten diese auf mein Bemühen, die Situation der Demonstration rückblickend zu beschreiben, hatten sie doch das spannungsvolle Geschehen hautnah miterlebt.

Wir drei befanden uns, so begann ich meine Ausführungen, auf einer «öffentlichen Bühne», während die anderen zuschauten. Dies erzeugt natürlich enormen Stress, auch wenn man sich freiwillig für eine solche Demonstration meldet. Für einen Moment schlüpfte ich in die Rolle der Teilnehmerin mit den Worten, dass ich eventuell Scham empfinden könnte, hier in dieser hervorgehobenen Position zu stehen, in aller Öffentlichkeit sichtbar zu sein und dabei über mich und meinen Körper zu erzählen. Natürlich würde es mir auch, wenn ich über meinen Bauch- Beckenraum reden wollte, die Sprache verschlagen können, wüsste ich doch, dass dieser Bereich mit sehr persönlichen, intimen oder gar sexuellen Gefühlen besetzt sein könnte. Beide Chinesinnen schienen richtig aufzuatmen, bestätigten die Plausibilität meiner Ausführungen und konnten sich vorstellen, Ähnliches zu empfinden, wie ich es gerade berichtet hatte. Mir ging es in dem Moment nicht um eine erneute Rückmeldung der Teilnehmerin, die sich bereit erklärt hatte, die Übung zu machen, sondern darum, allen im Workshop das szenische Erleben von gemeinsamem, vertrauensvollem Kontakt über das Erleben und Verstehen desselben nahezubringen. In dem Moment war die erneute persönliche Berichterstattung durch die Teilnehmerin über das, was sie im Körper erlebt hatte, nicht mehr vonnöten. Wir verabschiedeten uns also und die Teilnehmerin ging zurück zu ihrem Platz. Wenig später bemerkte ich mit grossem Erschrecken, dass sie den Raum verliess, obwohl der Workshop noch gar nicht zu Ende war.

Ohne weiter auf das Geschehen bei der Demonstrationsübung einzugehen, bezog ich mich nun auf das Verlassen des Raumes durch die Teilnehmerin. Spontan und persönlich erzählte ich von meinem Erleben dieser Situation, von meiner Bestürzung über die tiefgreifende Auswirkung der an sich kleinen Demonstrationsübung. Ich schilderte natürlich auch meine Erleichterung, zumal ich den Schritt der Teilnehmerin auch als einen Selbstschutz verstanden hatte. 
Mir war wichtig, das Geschehen auf mein allgemeines Workshop-Thema «Einführung in Körpersprache und nonverbale Kommunikation» zu beziehen. Körper, so führte ich aus, ist immer gefühlter, erlebter und erlebbarer Körper. Entsprechend der Situation und der jeweiligen Beziehung zu den anwesenden Menschen kommt es zu einem graduell unterschiedlichen Bewusstsein hierüber. Je intimer, je schambesetzter der erlebte Körper ist, desto eher beginnt sich hierüber ein Mantel der Wort- und Sprachlosigkeit zu legen bzw. legen zu können. Mit dem erlebten und erlebbaren Körper zu arbeiten, berührt immer - auch wenn man es vermeiden möchte - diese Dimension in der Beziehung. Ein zweiter Aspekt, so führte ich aus, ist «der Körper in der Öffentlichkeit». Körperlich ist man immer sichtbar, auch wenn man sich verbergen will. Entweder füblt man sich so, auch wenn andere dies nicht merken sollten, ist also sich selbst gegenüber mit diesen Gefühlen «sichtbar» und "öffentlich»; oder man ist anderen gegenüber faktisch sichtbar, tatsächlich öffentlich. Dies kann man nie vermeiden. Sich mit dem Körper zu beschäftigen, sei es in einer Beziehung oder in Therapie oder Beratung, induziert immer das unbewusste Erleben, sich wie auf einer öffentlichen Bühne ungeschützt zu erleben. Natürlich ist dies mit Scham verbunden. Diese kann in der Arbeit mit dem Körper als Therapeut oder Berater nie vermieden werden.

Beide von mir beschriebenen Aspekte waren den Chinesen erfahrungsmässig sowie kulturell neu, sodass sich eine lebhafte Diskussion hierüber ergab. Mit Verwunderung, aber auch mit einem gewissen Erschrecken führten sie sich vor Augen, dass Schamerleben immer auch eine Körper-zu-Körper-Kommunikation ist, auch wenn man nur miteinander spreche. Man selbst als Therapeut oder Berater ist daher immer mit im Spiel, bezieht man sich doch, ob man es will oder nicht, immer gerade auch nonverbal oder paraverbal auf den Klienten. Das Gleiche gilt natürlich auch für den zwischenmenschlichen Umgang im nicht-professionellen Bereich.

Das Gespräch wirkte wie eine Ermutigung dazu, sich nun auch persönlich in Kleingruppen über die eigene Erfahrung mit Intimität, Scham und Sexualität auszutauschen. Auch heute noch bin ich gelegentlich über das plötzliche Verlassen des Raumes durch die Teilnehmerin bestürzt und beschämt, bin ich doch selbst «Mit-Verursacher» dieser Szene gewesen. Ich habe diese Reaktion natürlich nicht vermeiden können. Unabhängig davon konnten alle Beteiligten hautnah und persönlich miterleben, wie es sich anfühlt, sich in der Öffentlichkeit körperlich zu zeigen und gesehen zu werden. Man könnte fast sagen, die emotionale «Härte» solcher Situationen bestehe darin, diesem Geschehen nie entgehen zu können, auch wenn man bemüht ist, es zu tun. Man könnte auch sagen, je mehr man die Scham zu unterdrücken bemüht ist, desto schmerzlicher wird sie spürbar. Scham ist nämlich das Gefühl, dem man in einem solchen Moment des Erlebens nie entgehen kann.

Über die selbst szenisch erlebte Scham Chinesen gegenüber zu sprechen, erfordert von mir als Westler einen guten Kontakt in der jeweiligen Situation in China und kulturelles Fingerspitzengefühl, schweigen Chinesen doch anfangs regungslos lauschend, wie hypnotisiert oder kindlich gehemmt, mit einem atemlos wirkenden Blick.

\section{Meine ständigen Begleiter - oder: Ménage-à-quatre}

Der Alltag auf meiner ethnologischen Wanderung war recht pragmatisch und oft gar nicht so kompliziert. Wenn ich mein Hotelzimmer verliess, traf ich im Frühstücksraum Chinesen; im Aufzug ebenso. Wenn ich die Strasse betrat, konnte ich gar nicht anders, als Chinesen zu sehen. Und wenn ich mit jemandem über etwas sprach, was mich gerade beschäftigte, ging es dabei immer auch um Chinesen, wie sie sich sehen und wie ich sie sehe. Der pragmatische und heuristische Zugang sowie meine spielerisch-kreative Grundhaltung erleichterten es mir, das erfinderische Kopfkino, meine kreativen Gedankenspielereien dabei einzudämmen oder gar auszuschalten. Gerade dann, und das erinnere ich noch sehr gerne, erfasste mich ein tiefer Spass, eine Freude, eine Lust mittendrin zu sein, mitzumachen, eben teilzunehmen in dem jeweiligen Situationsraum, in dem ich mich befand. Immer hatte ich dabei drei ständige Begleiter, sodass es immer vier Anwesende gab:

$>$ mich selbst mit meinem Wahrnehmungsapparat

$>$ den/die jeweiligen Chinesen, mit dem/denen ich befasst war

$>$ mein Begleiter bzw. Übersetzer

$>$ meinen emotionalen Resonanzkörper

Da sich die Beziehung also zwischen vier Beteiligten entwickelt, wähle ich hierfür die Bezeichnung «Ménageà-quatre». Diese verstehe ich als transkulturelle Variation bzw. Erweiterung einer Ménage-à-trois. Letztere bezieht alle Drei emotional mit ein. Im Unterschied hierzu verstehe ich die Ménage-à-quatre als eine prinzipiell offene Beziehung von vier Beteiligten. Die Offenheit entsteht durch den Umstand, dass stets maximal drei Personen miteinander kommunizieren. Die vierte Position bleibt offen, wirkt somit wie ein strukturell verunsicherndes Element. Die Ménage-à-quatre ist also durch strukturelle Irritation, kommunikative Toleranz sowie durch ein prinzipielles «implizites Geheimnis» gekennzeichnet:

$>$ Strukturelle Irritation ergibt sich aus dem Umstand, dass stets nicht alle vier Beteiligten gleichzeitig aktiv sind. Es gibt also immer eine unsichere, unkalkulierbare Komponente, sozusagen einen strukturellen blinden Fleck.

> Kommunikative Toleranz oder auch kommunikative «Unfertigkeit» bezieht sich auf das jeweils offene Element (blinder Fleck) in der Kommunikation unter den vier Beteiligten. Wenn zwei oder drei miteinander kommunizieren, endet dieser Austausch letztendlich offen, gibt es doch stets einen Vierten, an der jeweiligen Szene nicht Einbezogenen, der sich dann zu Wort meldet oder auf den man sich bezieht. Und auch der 
hierdurch entstehende Austausch findet dann in der Regel nur unter maximal drei Beteiligten statt.

> Prinzipiell wirkt daher immer auch ein nicht greifbarer, nicht erkennbarer Aspekt, der den Charakter eines impliziten Geheimnisses induziert.

Während ich mir in dieser Konstellation anfangs doch eher einsam, hilflos und unwissend vorkam, begann ich die Gesellschaft zu viert, diese Ménage-à-quatre, bald zu geniessen.

\section{Alles oder Nichts - oder: Anstelle eines Epilogs}

Noch gut erinnere ich mich an die Begegnung mit einer französischen Ethnologin, die 1979 auf der indonesischen Insel Sumba forschte. Als ich sie vor Ort traf, war sie kaum noch ihrer Muttersprache mächtig. Sie wirkte auf mich scheu, zurückhaltend und auch subtil genervt, so als wollte sie unser Gespräch schnellstmöglich wieder beenden. Sie betonte, dass sie voll und ganz in die Lebenswelt der Menschen auf Sumba eingetaucht sei. Sie hätte dies so tun müssen. Sie wollte die Lebenswelt kennenlernen, die Düfte riechen, die Klänge der Instrumente in sich selbst schwingen fühlen, natürlich auch die Sprache verstehen und ihre Melodie singend sprechen lernen. Natürlich war sie auch fasziniert von der Chance, tiefe ethologische Einblicke in die Art der Lebensgestaltung der Menschen auf Sumba zu gewinnen. Ich fragte mich damals, wie sie denn wohl wieder in ihre eigene Kultur, in ihre Heimat zurückkehren könnte, wenn sie sich doch so dieser magischen und so fremden Lebenswelt hingegeben hatte.

Vielleicht ist dies ja die (notwendige) Verlockung, als Psychotherapeut transkulturell tätig zu werden, nämlich anfangs eine Grundhaltung zu erwerben, indem man in die fremde Kultur eintaucht. Eine Haltung, die eine unverzichtbare Voraussetzung ist, mit Menschen, die aus einer fremden Kultur kommen, überhaupt therapeutisch arbeiten zu können.

\section{Literatur}

Geertz, C. (2003). Dichte Beschreibung. Beiträge zum Verstehen kultureller Systeme. Frankfurt a. M.: Suhrkamp.

Geuter, U. (2015). Körperpsychotherapie. Grundriss einer Theorie für die klinische Praxis. Berlin, Heidelberg: Springer.

Honer, A. (2008). Lebensweltanalyse in der Ethnographie. In U. Flick, E. v. Kardorff \& I. Steinke (Hrsg.), Qualitative Forschung - Ein Handbuch (S. 194-204; 6. Aufl.). Hamburg: Rowohlt.

Klüwer, C. (1974). Kinder und Jugendliche im psychosozialen Spannungsfeld. Unveröffentlichtes Vorlesungsmanuskript der Vorlesungsreihe am sozialpsychologischen Institut der Ruhruniversität Bochum.

Leonard, M. (2018). Der Alchimist. Süddeutsche Zeitung, 27.04.2018.

Lindner, R. (2004). Walks on the wild side. Eine Geschichte der Stadtforschung. Frankfurt a. M., New York: Campus.

Lorenzer, A. (1972). Zur Begründung einer materialistischen Sozialisationstheorie. Frankfurt: Suhrkamp.

Nazarkiewicz, K. \& Krämer, G. (2012). Handbuch interkulturelles Coaching. Göttingen: Vandenhoeck \& Ruprecht.

Northoff, G. (2015). Wie kommt die Kultur in den Kopf? Berlin, Heidelberg: Springer.
Ricoeur, Paul (1973). Hermeneutik und Strukturalismus. München: Kösel.

Schatz, D.S. (2002). Klassifikation des Körpererlebens und körperpsychotherapeutische Hauptströmungen. Psychotherapeut, 44, $47-87$.

Simon, F. B. (2010 [1997]). Die Kunst, nicht zu lernen. Und andere Paradoxien in Psychotherapie, Management, Politik (5. Aufl.). Heidelberg: Carl-Auer-Verlag.

Sollmann, U. (2018). Begegnung im Reich der Mitte - mit psychologischem Blick unterwegs in China. Gießen: Psychosozial-Verlag.

\section{Development and significance of a transcultural attitude as a body-psychotherapist shown on the example of China}

For the author, presenting Body-Psychotherapy to colleagues in China became an ethnological process of experience. Being part of the action in the various «lived-in-worlds» (professional, personal or privat) took shape as body-to-bodycommunication. Involved in this way and according to a professional naivety the author used a heuristic approach to dis-learn (of his own cultural pre-understanding) first (EntLernen). So he was able to generate a «knowledge» that was based on sensory, emotional and transcultural communication. Essentially the process was an open one. The author presumed the way of narration as the best to reproduce such a process of «sensed knowing» effectively, vivid and even professional, the form of a professional essay. The article illuminates several aspects of interplay of structure, space of «lived-in-worlds» and of the dynamic, colorful, complex and arbitrary self-regulation of people in these structures. Besides the article gives an insight in «ethnological instruments», that is useful for transcultural communication.

Key-Words: Body-Psychotherapy, China, ethnology, narration, patterns of behavior, transcultural communication.

\section{Sviluppo e significato di un atteggiamento transculturale come psicoterapeuta (del corpo) nell'esempio della Cina. Un saggio etnoanalitico}

Presentare ai colleghi in Cina i concetti di psicoterapia del corpo, rappresenta per l'autore un processo esperienziale etnologico. Ciò implicito, l'autore ha utilizzato un percorso euristico purché in modo corrispondente a un processo di de-apprendimento (la propria comprensione culturale) ci si occupasse di generare un «sapere» basato sull'esperienza dei sensi, che soprattutto si fondasse anche sulla comunicazione sensoriale, emozionale nonché transculturale. Purché il processo fosse principalmente aperto. Al fine di restituire tale processo del «sensed knowing» in modo efficace, plastico ma anche professionale, a questo punto all'autore sembra adatto il mezzo della narrazione, vale a dire la forma di un saggio professionale. Nel contributo si fa esperienza da una parte dell'interazione in Cina tra struttura, campi di vita nonché dell'autoregolazione variopinta, stratificata, dinamica, ostinata degli uomini a livello locale. D'altra parte l'articolo offre sguardi alla «valigia di strumenti» etnologica, che nella comunicazione transculturale può dare supporto, senso e risultare utile dal punto di vista pratico. 
Parola chiave: psicoterapia del corpo, Cina, etnologia, narrazione, modello di comportamento, comunicazione transculturale

\section{Der Autor}

Ulrich Sollmann, Dipl.rer.soc., Körperpsychotherapeut (Bioenergetische Analyse, Gestaltpsychotherapie), Mitglied der Deutsch-Chinesischen Akademie für Psychotherapie (DCAP),
Berater und Coach in Wirtschaft und Politik, Publizist, Blogger. Arbeitet in freier Praxis in Bochum sowie regelmässig in China. Vortrags- und Lehrtätigkeit (u.a. Scholarship as guest professor at Shanghai University of Political Science and Law).

\section{Kontakt}

info@sollmann-online.de 